\title{
Bimagnetic nanoparticles with enhanced exchange coupling and energy products
}

\author{
Vikas Nandwana, Girija S. Chaubey, Kazuaki Yano, Chuan-bing Rong, and J. Ping Liu ${ }^{\text {a) }}$ \\ Department of Physics, University of Texas at Arlington, Arlington, Texas 76019, USA
}

(Received 27 August 2008; accepted 12 November 2008; published online 6 January 2009)

\begin{abstract}
Bimagnetic $\mathrm{FePt} / \mathrm{Fe}_{3} \mathrm{O}_{4}$ nanoparticles with core/shell or heterodimer structure have been prepared using a sequential synthetic method. The dimension of both $\mathrm{FePt}$ and $\mathrm{Fe}_{3} \mathrm{O}_{4}$ was tuned by varying the synthesis parameters. The as-synthesized bimagnetic nanoparticles were superparamagnetic at room temperature. After being annealed in a reducing atmosphere, the $\mathrm{FePt} / \mathrm{Fe}_{3} \mathrm{O}_{4}$ bimagnetic nanoparticles were converted to a hard magnetic nanocomposite with enhanced energy products due to the exchange coupling between the hard and soft magnetic phases. It was found that the exchange coupling in nanocomposites made from the core/shell nanoparticles is stronger than that from the heterodimer nanoparticles. By tuning the dimensions of the $\mathrm{FePt}$ and $\mathrm{Fe}_{3} \mathrm{O}_{4}$ phases, the energy product up to 17.8 MGOe was achieved in the annealed nanocomposites, which is $36 \%$ higher than the isotropic single-phase FePt counterpart. (C) 2009 American Institute of Physics.
\end{abstract}

[DOI: $10.1063 / 1.3054441]$

\section{INTRODUCTION}

Conventional techniques are used to prepare nanocomposite magnets such as melt-spinning produce materials with wide grain size distribution in the magnets, which is not favorable to effective exchange coupling. An alternative bottom-up approach to fabricate exchange-coupled nanocomposite magnets has been recently developed, which starts from nanoparticle synthesis. ${ }^{1-9}$ With this approach, two different methodologies can be used to produce nanoscale composite magnets. The first method is to blend hard and soft magnetic nanoparticles that are synthesized separately to form nanoscale hard/soft nanoparticle assemblies. ${ }^{2,5,6}$ This approach requires precise control over the mass ratio and diameters of both the hard and soft magnetic nanoparticles, and the mixed nanoparticle assembly conditions have to be controlled carefully to achieve homogeneous distribution of different phases. With the second methodology, bimagnetic nanoparticles containing hard and soft phases are synthesized so that no blending process is necessary because each particle is a nanocomposite. ${ }^{3,4,7}$ For instance, FePt-based nanocomposite magnets can be produced by a two-step synthesis route, where FePt seeds were first prepared and then the seeds were coated with ferrite layers. This method is more convenient in controlling dimensions of different components in order to achieve desired intimate interphase contact and morphology homogeneity. Exchange-coupled hard/soft bimagnetic nanoparticles are also proposed for future high density recording media over single hard phase nanoparticles since their coercivity is within the field limit imposed by writing head. ${ }^{10}$ In this study we report synthesis of $\mathrm{FePt} / \mathrm{Fe}_{3} \mathrm{O}_{4}$ bimagnetic nanoparticles by a one-pot sequential method in which the soft magnetic $\mathrm{Fe}_{3} \mathrm{O}_{4}$ phase was grown on FePt nanoparticles directly, either coated or attached to FePt nanoparticles in a controlled manner. The di-

${ }^{\text {a)} E l e c t r o n i c ~ m a i l: ~ p l i u @ u t a . e d u . ~}$ mension of the FePt as well as the $\mathrm{Fe}_{3} \mathrm{O}_{4}$ phases can be finely tuned by adjusting the reaction parameters. After being annealed in a reducing atmosphere, the $\mathrm{FePt} / \mathrm{Fe}_{3} \mathrm{O}_{4}$ bimagnetic nanoparticles transformed to an exchange-coupled $\mathrm{FePt} / \mathrm{Fe}_{3} \mathrm{Pt}$ hard magnetic nanocomposite with enhanced energy products.

\section{EXPERIMENTAL}

All the reagents used in this synthesis are commercially available and were used as received without further purification. All the reactions were carried out using standard schlenk line technique. Two stage heating strategies were used to prepare bimagnetic $\mathrm{FePt} / \mathrm{Fe}_{3} \mathrm{O}_{4}$ nanoparticles. First, FePt nanoparticles were synthesized by chemical reduction in platinum acetylacetonate $\left[\mathrm{Pt}(\mathrm{acac})_{2}\right]$ and thermal decomposition of iron pentacarbonyl $\left[\mathrm{Fe}(\mathrm{CO})_{5}\right]$ in the presence of surfactants, oleic acid, and oleyl amine. In brief, $0.5 \mathrm{mmol}$ of platinum acetylacetonate was added to $125 \mathrm{ml}$ flask containing a magnetic stir bar and mixed with $10 \mathrm{ml}$ of octyl/benzyl ether. After purging with argon for $30 \mathrm{~min}$ at room temperature, the flask was heated up to $120^{\circ} \mathrm{C}$ until the platinum precursor dissolved completely in the solvent. Then, 1.0 mmol of iron pentacarbonyl and a designated amount of oleic acid and oleyl amine were added at $120^{\circ} \mathrm{C}$. The flask was then heated to $230{ }^{\circ} \mathrm{C}$ for $1 \mathrm{~h}$ and then refluxed for $1 \mathrm{~h}$ before cooling down to $205{ }^{\circ} \mathrm{C}$ for further reaction. The size of the FePt nanoparticles was tuned by changing the surfactant to platinum precursor ratio. ${ }^{11}$

To grow $\mathrm{Fe}_{3} \mathrm{O}_{4}$ on the $\mathrm{FePt}$ nanoparticles, a designated amount of iron pentacarbonyl was injected into the flask at $205^{\circ} \mathrm{C}$ and the temperature of the reaction mixture was raised to $290{ }^{\circ} \mathrm{C}$ for $30 \mathrm{~min}$ before cooling down to ambient temperature. The black product was precipitated by adding ethanol and separated by centrifugation and redispersed in hexane. By controlling the molar amount of Fe precursor in the second step, the dimension of $\mathrm{Fe}_{3} \mathrm{O}_{4}$ phase was readily tuned. The amount of Fe and Pt precursors in the first step 


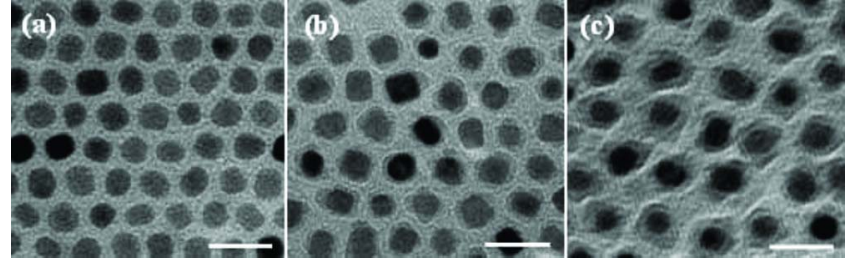

FIG. 1. (Color online) TEM images of the as-synthesized (a) $7 \mathrm{~nm} \mathrm{FePt}$ nanoparticles, (b) $7 \mathrm{~nm}$ FePt nanoparticles coated with $1 \mathrm{~nm}$, and (c) $7 \mathrm{~nm}$ $\mathrm{FePt}$ nanoparticles coated with $3 \mathrm{~nm} \mathrm{Fe}_{3} \mathrm{O}_{4}$ shell (scale bar is $20 \mathrm{~nm}$ ).

was always kept constant. The morphology of these bimagnetic $\mathrm{FePt} / \mathrm{Fe}_{3} \mathrm{O}_{4}$ nanoparticles was changed by merely changing the solvent in the reaction. When benzyl ether was used as the solvent, core/shell nanoparticles were obtained, while in the case of octyl ether, heterodimer nanoparticles were observed.

After being washed in ethanol three or more times, the bimagnetic nanoparticles were dispersed in hexane and stored in glass bottles under refrigeration. Samples for magnetic characterization were prepared by depositing a drop of the final hexane dispersion on a $3 \times 3 \mathrm{~mm}^{2}$ silicon substrate, evaporating the solvent at room temperature, and further drying in vacuum, which led to the formation of $\mathrm{FePt} / \mathrm{Fe}_{3} \mathrm{O}_{4}$ nanoparticle-assembled thin films. The samples were then annealed at $650{ }^{\circ} \mathrm{C}$ for $1 \mathrm{~h}$ under the flow of forming gas $\left(\mathrm{Ar}+7 \% \mathrm{H}_{2}\right)$ in a tube furnace. The transmission electron microscopy (TEM) images were recorded on a JEOL 1200 EX electron microscope at an accelerating voltage of $120 \mathrm{kV}$. Powder X-ray diffraction (XRD) spectra were recorded with a $\mathrm{Cu} K \alpha$ x-ray source $(\lambda=1.5405 \AA)$. The magnetic hysteresis measurements were carried out by using superconducting quantum interference device magnetometer with magnetic field up to $7 \mathrm{~T}$. The composition analysis was done by energy dispersive $\mathrm{x}$-ray spectroscopy.

\section{RESULTS AND DISCUSSION}

Figures 1 and 2 show TEM images of core/shell structured $\mathrm{FePt} / \mathrm{Fe}_{3} \mathrm{O}_{4}$ nanoparticles with different core and shell dimensions. For each particle, the darker region is the FePt core and the lighter part is the $\mathrm{Fe}_{3} \mathrm{O}_{4}$ shell. The different

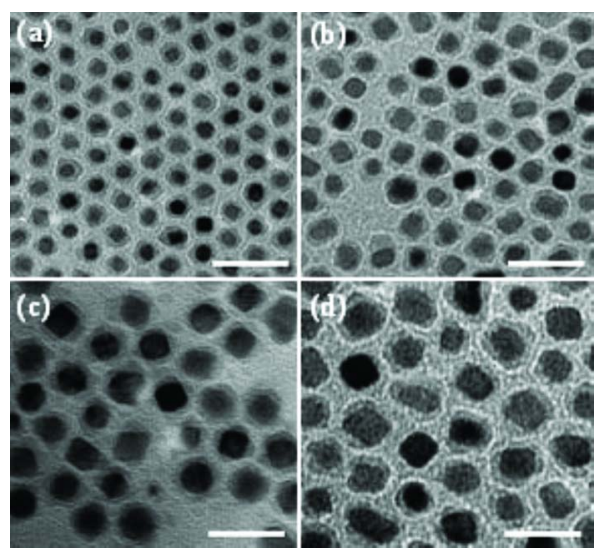

FIG. 2. (Color online) TEM images of as-synthesized $\mathrm{FePt} / \mathrm{Fe}_{3} \mathrm{O}_{4}$ (a) 4 $\mathrm{nm} / 2 \mathrm{~nm}$, (b) $6 \mathrm{~nm} / 1 \mathrm{~nm}$, (c) $8 \mathrm{~nm} / 2 \mathrm{~nm}$, and (d) $8 \mathrm{~nm} / 3 \mathrm{~nm}$ core/shell nanoparticles (scale bar is $20 \mathrm{~nm}$ ).

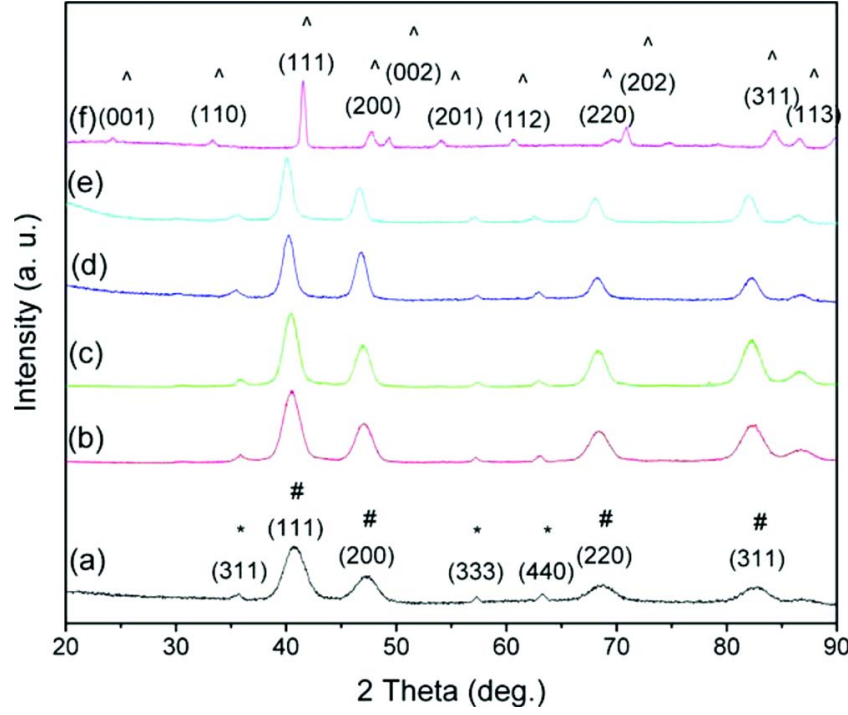

FIG. 3. (Color online) XRD of the as-synthesized $\mathrm{FePt} / \mathrm{Fe}_{3} \mathrm{O}_{4}$ core/shell nanoparticles with FePt(\#) core diameter (a) $4 \mathrm{~nm}$, (b) $5 \mathrm{~nm}$, (c) $6 \mathrm{~nm}$, (d) 7 nm, (e) $8 \mathrm{~nm}$ with $1 \mathrm{~nm} \mathrm{Fe}_{3} \mathrm{O}_{4}\left({ }^{*}\right)$ shell thickness and (f) $\mathrm{Fe}_{3} \mathrm{Pt} / \mathrm{FePt}(\wedge)$ nanocomposite after annealing of $8 \mathrm{~nm} \mathrm{FePt} / 1 \mathrm{~nm} \mathrm{Fe} \mathrm{O}_{4}$ after annealing at $650{ }^{\circ} \mathrm{C}$ for $1 \mathrm{~h}$.

contrasts between these two regions are due to the different electron penetration efficiencies of the metallic FePt and the oxide $\mathrm{Fe}_{3} \mathrm{O}_{4}$. It is clear from Fig. 1 that the monodispersed $\mathrm{FePt}$ nanoparticles are homogeneously coated with $\mathrm{Fe}_{3} \mathrm{O}_{4}$ shells with controllable shell thickness. The reaction conditions have to be well controlled in order to prevent any separate nucleation of $\mathrm{Fe}_{3} \mathrm{O}_{4}$ nanoparticles, especially when the shell thickness is increased. Figure 2 shows TEM images of core/shell structured $\mathrm{FePt} / \mathrm{Fe}_{3} \mathrm{O}_{4}$ nanoparticles with a systematic control in size of the FePt core between 4 and $8 \mathrm{~nm}$ and $\mathrm{Fe}_{3} \mathrm{O}_{4}$ shell thickness from 1 to $3 \mathrm{~nm}$. It is clearly seen from Figs. 1 and 2 that the monodispersed FePt nanoparticles with different sizes are homogeneously coated with $\mathrm{Fe}_{3} \mathrm{O}_{4}$ shells.

Figures 3(a)-3(e) show XRD patterns of the assynthesized $\mathrm{FePt} / \mathrm{Fe}_{3} \mathrm{O}_{4}$ core/shell nanoparticles with $\mathrm{FePt}$ core sizes of 4, 5, 6, 7, and $8 \mathrm{~nm}$ with $1 \mathrm{~nm} \mathrm{Fe}{ }_{3} \mathrm{O}_{4}$ shells. The patterns contain two sets of peaks, with one set matching with the disordered fcc FePt and the other with cubic spinel structured $\mathrm{Fe}_{3} \mathrm{O}_{4}$. It can also be seen that the width of (111) peak of FePt decreases as the FePt size increases. The size values of the FePt nanoparticles calculated using Scherrer's formula $^{12}$ are consistent with what are observed from the TEM images.

Figure 4 shows TEM images of a different morphology of bimagnetic $\mathrm{FePt} / \mathrm{Fe}_{3} \mathrm{O}_{4}$ nanoparticles. As mentioned earlier, when octyl ether was used as the solvent of the chemical solution synthesis, heterodimer $\mathrm{FePt} / \mathrm{Fe}_{3} \mathrm{O}_{4}$ nanoparticles with $\mathrm{FePt}$ size of $8 \mathrm{~nm}$ and $\mathrm{Fe}_{3} \mathrm{O}_{4}$ dimensions of 5, 8, and 10 $\mathrm{nm}$ can be synthesized. It is interesting to know the mechanisms of formation of the core/shell and heterodimer structured nanoparticles. Since FePt nanoparticles were already presented in the reaction mixture, $\mathrm{Fe}_{3} \mathrm{O}_{4}$ nucleated preferably on surface of the FePt seeds rather than nucleating separately to form independent $\mathrm{Fe}_{3} \mathrm{O}_{4}$ nanoparticles. The final morphology depends on whether the FePt surface allows only a 


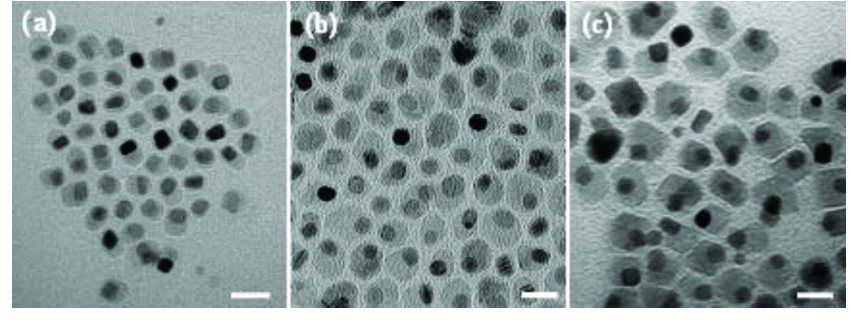

FIG. 4. (Color online) TEM images of the as-synthesized $\mathrm{FePt} / \mathrm{Fe}_{3} \mathrm{O}_{4}$ heterodimer nanoparticle with $8 \mathrm{~nm}$ FePt attached with (a) $5 \mathrm{~nm}$, (b) $8 \mathrm{~nm}$, and (c) $10 \mathrm{~nm} \mathrm{Fe}{ }_{3} \mathrm{O}_{4}$ (scale bar is $20 \mathrm{~nm}$ ).

single nucleation site or multiple ones. As suggested by Yu et al., ${ }^{13}$ free electrons in the solution may also catalyze the $\mathrm{Fe}_{3} \mathrm{O}_{4}$ nucleation. Solvent such as benzyl ether contains aromatic ring, which is good free electron donors. Their coordination to the FePt nanoparticles may replenish the electronic deficiency on the latter, consequently allow additional nuclei to form on the FePt surface, which can grow and eventually coalesce to form core/shell structures. In contrast, solvent with largely saturated hydrocarbon chains such as octyl ether is not effective electron donors, and once a single $\mathrm{Fe}_{3} \mathrm{O}_{4}$ nucleus is present further nucleation may not be possible without electron donation from the solvent. Further growth of $\mathrm{Fe}_{3} \mathrm{O}_{4}$ from a single site then can lead to formation of a heterodimer nanoparticle.

It is even more interesting to know the magnetic property change upon the morphology change. The assynthesized $\mathrm{FePt}$ and $\mathrm{FePt} / \mathrm{Fe}_{3} \mathrm{O}_{4}$ nanoparticles were found to be superparamagnetic at room temperature due to the low magnetocrystalline anisotropy of disordered fcc FePt. To obtain hard magnetic nanocomposite, the as-synthesized $\mathrm{FePt} / \mathrm{Fe}_{3} \mathrm{O}_{4}$ nanoparticles were annealed in a reducing atmosphere $\left(7 \% \mathrm{H}_{2}+93 \% \mathrm{Ar}\right)$ at $650{ }^{\circ} \mathrm{C}$ for $1 \mathrm{~h}$. As shown in Fig. 3(f), the XRD patterns confirmed the developed $\mathrm{FePt} / \mathrm{Fe}_{3} \mathrm{Pt}$ nanocomposite magnets being annealed at $650{ }^{\circ} \mathrm{C}$. The appearance of FePt $L 1_{0}$ peaks in XRD patterns showed the transformation of FePt phase from disordered fcc FePt struc- ture to the magnetically hard $L 1_{0}$ structure. The $\mathrm{Fe}_{3} \mathrm{O}_{4}$ was transformed into $\mathrm{Fe}_{3} \mathrm{Pt}$ magnetic soft phase during the reductive annealing. The similar works have been reported for the $\mathrm{FePt} / \mathrm{Fe}_{3} \mathrm{O}_{4}$ mixtured $^{9}$ and bricklike ${ }^{14}$ nanoparticles systems.

During the first step of the reaction, the composition of $\mathrm{Fe} / \mathrm{Pt}$ in FePt nanoparticles was kept $\mathrm{Fe}_{52} \mathrm{Pt}_{48}$ in all experiments since it is an optimized composition to obtain highest coercivity from FePt nanoparticles. ${ }^{3}$ The ratio of $\mathrm{Fe} / \mathrm{Pt}$ in core/shell and heterodimers $\mathrm{FePt} / \mathrm{Fe}_{3} \mathrm{O}_{4}$ nanoparticles was higher than that of $\mathrm{FePt}$ nanoparticles due to the presence of $\mathrm{Fe}_{3} \mathrm{O}_{4}$ phase. For example, for $8 \mathrm{~nm} / 1 \mathrm{~nm}$ and $8 \mathrm{~nm} / 3 \mathrm{~nm}$ as-synthesized $\mathrm{FePt} / \mathrm{Fe}_{3} \mathrm{O}_{4}$ core/shell nanoparticles, the composition of $\mathrm{Fe} / \mathrm{Pt}$ was found $61 / 39$ and 74/26, respectively. The ratio was found to be the same after annealing in the $\mathrm{FePt} / \mathrm{Fe}_{3} \mathrm{Pt}$ nanocomposite particle assemblies. As a comparison, annealing an assembly containing iron-rich $\mathrm{Fe}_{65-70} \mathrm{Pt}_{30-35}$ alloy nanoparticles led to materials that are magnetically much softer $\left(H_{c}\right.$ less than $\left.1 \mathrm{kOe}\right)$. This suggests that the hard magnetic property of the annealed hard/soft nanoparticle assembly originates from exchange-coupled hard and soft phases, not from the homogeneous iron-rich FePt alloy. Determination of the ratio of Fe/Pt in FePt and $\mathrm{Fe}_{3} \mathrm{Pt}$ phase in annealed core/shell nanoparticles requires accurate nanosized electronic beam diffraction analysis, is planned to do but has not yet been done.

Figures 5(a) and 5(b) show the hysteresis loops of annealed core/shell and heterodimer $\mathrm{FePt} / \mathrm{Fe}_{3} \mathrm{O}_{4}$ nanoparticles, respectively. The dimension of FePt core was kept same of 8 $\mathrm{nm}$ as an example. The hysteresis loop of the annealed single-phase FePt nanoparticles is also included in the figure for comparison purpose. It can be seen that the single-phase FePt nanoparticles have the highest coercivity but the lowest magnetization. There is an increase in magnetization while a decrease in coercivity as the dimension of the $\mathrm{Fe}_{3} \mathrm{O}_{4}$ shell was increased, for both the core/shell and the heterodimer nanoparticles. The soft-phase fraction in the core/shell particles with $2 \mathrm{~nm}$ shells is about the same as in the heterodimer particles with $8 \mathrm{~nm} \mathrm{Fe}_{3} \mathrm{O}_{4}$ attachments, both are
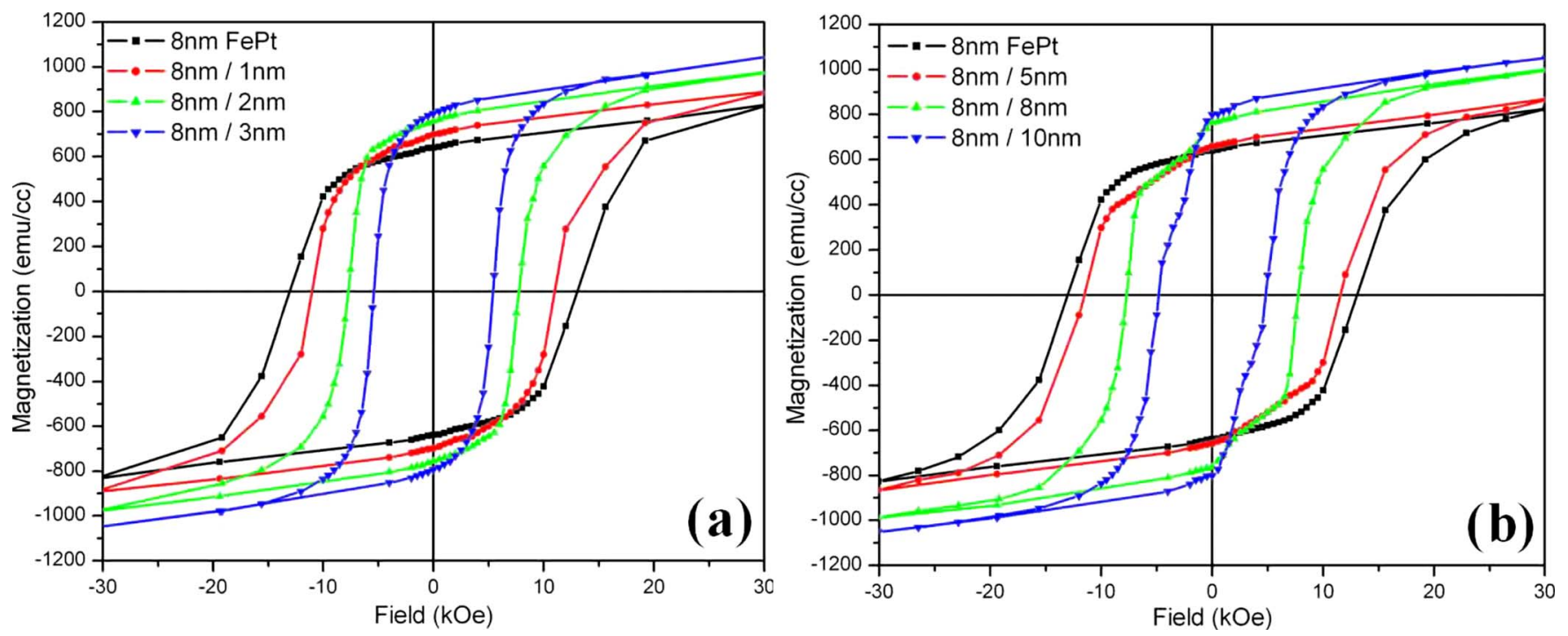

FIG. 5. (Color online) (a) Room-temperature hysteresis loops of annealed $\mathrm{FePt} / \mathrm{Fe}_{3} \mathrm{O}_{4}$ (a) core/shell nanoparticles with 8 nm FePt core and Fe $\mathrm{O}_{4}$ shell thickness increasing from 1 to $3 \mathrm{~nm}$. (b) Heterodimer nanoparticles with $8 \mathrm{~nm} \mathrm{FePt}$ and $\mathrm{Fe}_{3} \mathrm{O}_{4}$ from 5 to $10 \mathrm{~nm}$ at $650{ }^{\circ} \mathrm{C}$ for $1 \mathrm{~h}$. 

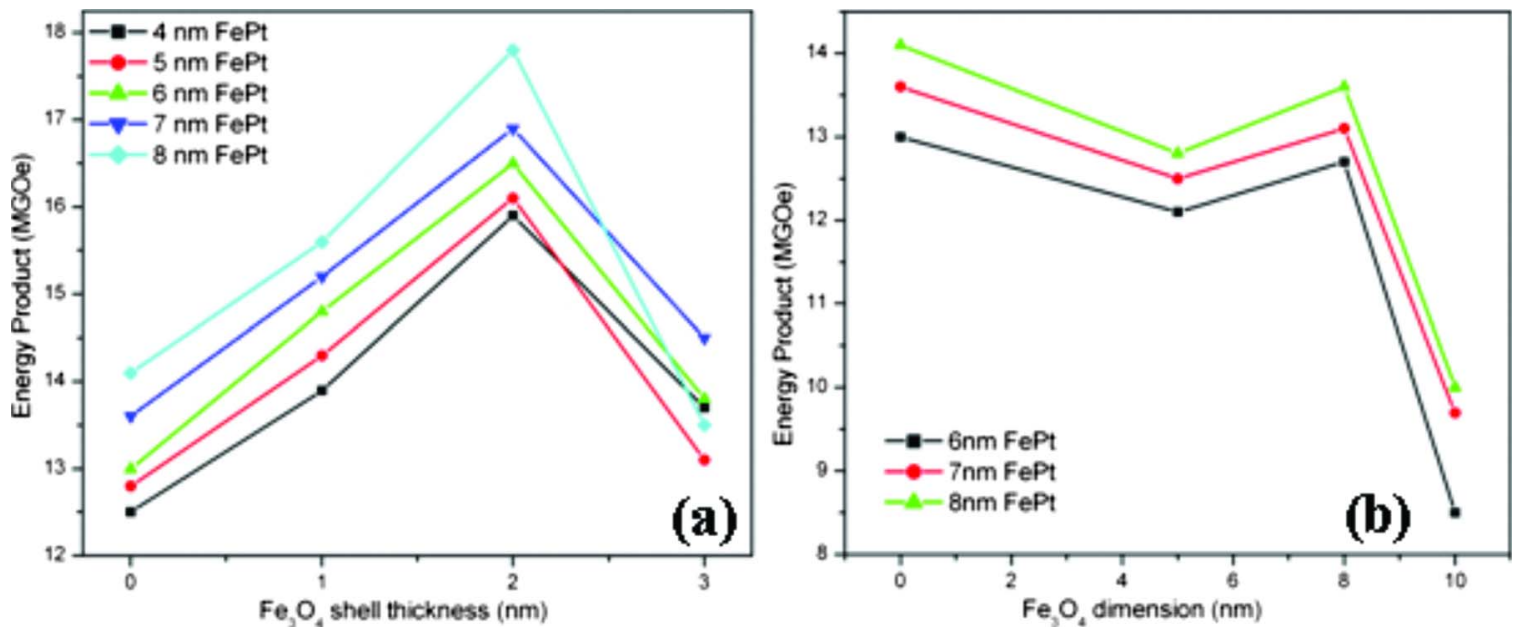

FIG. 6. (Color online) Variation in maximum energy product of the annealed $\mathrm{FePt} / \mathrm{Fe}_{3} \mathrm{O}_{4}$ (a) core/shell nanoparticles of different FePt core sizes (4-8 nm) and $\mathrm{Fe}_{3} \mathrm{O}_{4}$ shell thickness $(0-3 \mathrm{~nm})$ and (b) heterodimer nanoparticles of different sizes $\mathrm{FePt}(6-8 \mathrm{~nm})$ and $\mathrm{Fe}_{3} \mathrm{O}_{4}(0-10 \mathrm{~nm})$.

around $50 \%$ volume. The increase in magnetization is attributed to the formation of high-magnetization $\mathrm{Fe}_{3} \mathrm{Pt}$ soft phase during the annealing under the reducing atmosphere, as discussed above. However, the low magnetocrystalline anisotropy of the $\mathrm{Fe}_{3} \mathrm{Pt}$ phase leads to a decrease in coercivity of the nanocomposite magnets. The dependence of saturated magnetization and coercivity on the soft-phase fraction is quite similar to the numerical simulations, which were reported for the isotropic nanocomposite magnets. ${ }^{15,16}$

The energy product $\left[(B H)_{\max }\right]$ dependence on the soft phase dimension of the core/shell and heterodimer $\mathrm{FePt} / \mathrm{Fe}_{3} \mathrm{O}_{4}$ nanoparticles with different sizes is given in Fig. 6. It is found that the relation between $(\mathrm{BH})_{\max }$ and $\mathrm{Fe}_{3} \mathrm{O}_{4}$ dimension is not monotonous due to the fact that increasing the soft phase dimension will result in a trade-off between the magnetization and coercivity, as widely observed. In the case of core/shell particles, the $(B H)_{\max }$ was increased initially with increasing $\mathrm{Fe}_{3} \mathrm{O}_{4}$ shell thickness and decreased after certain $\mathrm{Fe}_{3} \mathrm{O}_{4}$ shell thickness. Our optimized conditions show that the highest energy product 17.8 MGOe was obtained in $8 \mathrm{~nm} / 2 \mathrm{~nm} \mathrm{FePt} / \mathrm{Fe}_{3} \mathrm{O}_{4}$ core/shell nanoparticles after being annealed at $650{ }^{\circ} \mathrm{C}$ for $1 \mathrm{~h}$. This value is $36 \%$ higher than the theoretical value of 13 MGOe for the nonexchange-coupled isotropic FePt single-phase material. Unlike core/shell particles, the $(B H)_{\max }$ from the heterodimer nanoparticles was not improved after being attached with $\mathrm{Fe}_{3} \mathrm{O}_{4}$. Instead of improvement, the $(\mathrm{BH})_{\max }$ was found even lower than FePt nanoparticles of all different sizes [Fig. 6(b)]. This is because energy products are not only dependent on the magnetization and coercivity but also on the hysteresis loop squareness. One can see kinks in the hysteresis loops from Fig. 5(b) for the heterodimer nanoparticles, indicating less effective exchange coupling between the hard and the soft phases.

To further understand the difference in exchangecoupling strength caused by the morphology, we measured the two groups of samples by Henkel plots ( $\delta m$ plots). The $\delta m$ values can be measured by $\delta m=m_{d}(H)-\left[1-2 m_{r}(H)\right]$, where $m_{d}$ is normalized demagnetization remanence and $m$ is the normalized isothermal magnetization remanence. ${ }^{17,18}$
Figure 7 shows the $\delta m$ curves of the core/shell and heterodimer nanoparticles with the same amount of soft phase after annealing at $650{ }^{\circ} \mathrm{C}$ for $1 \mathrm{~h}$. It is clearly seen that the positive $\delta m$ values were obtained in both the core/shell and heterodimer nanoparticles, indicating the exchange coupling between the hard and soft phases. Moreover, the $\delta m$ value of the annealed core/shell nanoparticles is substantially higher than that of the annealed heterodimer nanoparticles. This confirmed that the exchange coupling in the core/shell nanoparticles is stronger than that in the heterodimer nanoparticles. More interestingly, the curve for the heterodimer sample shows more negative portion, which is a measure for dipolar interaction. We observed that the negative portion is enhanced when large amount of soft phase is added to nanocomposite magnets. However, as we indicated that the soft phase fraction in these two samples is the same. This difference may be explained by a less homogeneous distribution of the soft phase in the nanocomposite magnets made from heterostructured nanoparticles.

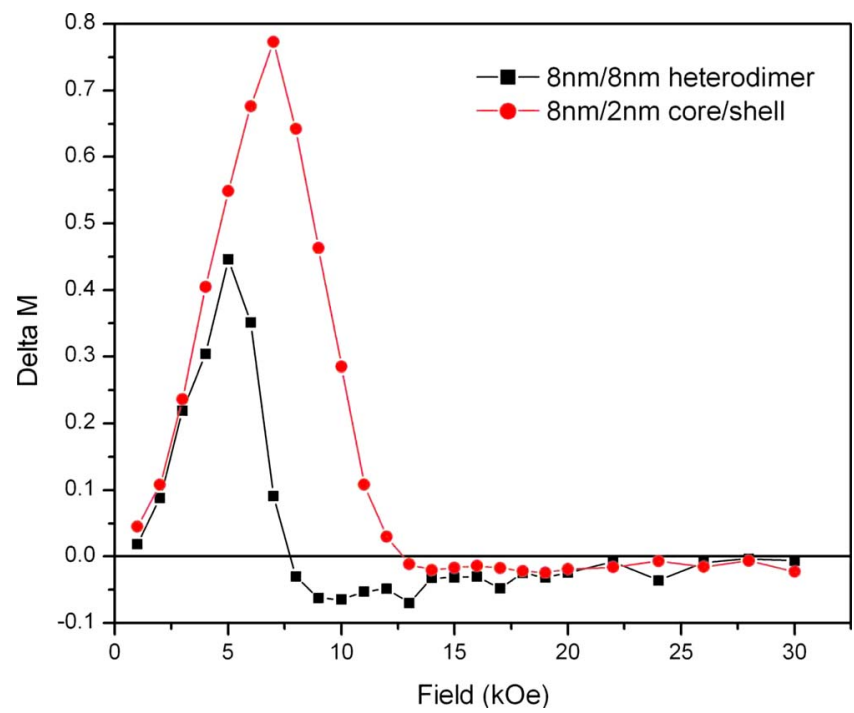

FIG. 7. (Color online) $\delta m$ curves of $8 \mathrm{~nm} / 2 \mathrm{~nm}$ core/shell and $8 \mathrm{~nm} / 8 \mathrm{~nm}$ heterodimer $\mathrm{FePt} / \mathrm{Fe}_{3} \mathrm{O}_{4}$ nanoparticles after annealing at $650{ }^{\circ} \mathrm{C}$ for $1 \mathrm{~h}$. 


\section{CONCLUSIONS}

$\mathrm{FePt} / \mathrm{Fe}_{3} \mathrm{O}_{4}$ bimagnetic nanoparticles with core/shell and heterodimer structures have been prepared in a one-pot sequential synthesis by reduction in platinum acetylacetonate and thermal decomposition of iron pentacarbonyl. The morphology type of the nanoparticles was controlled by changing solvent in the reactions. The size of $\mathrm{FePt}$ was tuned by changing surfactant to platinum precursor ratio, while the dimension of $\mathrm{Fe}_{3} \mathrm{O}_{4}$ phase was controlled by adjusting the amount of $\mathrm{Fe}(\mathrm{CO})_{5}$ precursor in the sequential step. After being annealed in a reducing atmosphere, the $\mathrm{FePt} / \mathrm{Fe}_{3} \mathrm{O}_{4}$ nanoparticles form a hard magnetic nanocomposite with enhanced magnetic properties, which are closely related to dimensions of the soft and hard phase components and their morphology. The heterodimer nanoparticles resulted in relatively poor magnetic properties compared to the core/shell nanoparticles due to insufficient exchange coupling. By optimizing the dimensions of the $\mathrm{FePt}$ and $\mathrm{Fe}_{3} \mathrm{O}_{4}$ in core/shell bimagnetic nanoparticles, energy product up to $17.8 \mathrm{MGOe}$ has been achieved, which is $36 \%$ higher than the theoretical value for isotropic single-phase FePt. These bimagnetic nanoparticles can be excellent building blocks for high performance nanocomposite magnets and high density recording media.

\section{ACKNOWLEDGMENTS}

This work was supported by ONR/MURI under Grant No. N00014-05-1-0497 and by the Center of Nanostructured
Materials and Characterization Center for Materials and Biology at the University of Texas at Arlington.

${ }^{1}$ D. J. Sellmyer, Nature (London) 420, 374 (2002).

${ }^{2}$ H. Zeng, J. Li, J. P. Liu, Z. L. Wang, and S. Sun, Nature (London) 420, 395 (2002).

${ }^{3}$ H. Zeng, J. Li, Z. L. Wang, J. P. Liu, and S. Sun, Nano Lett. 4, 187 (2004). ${ }^{4}$ H. Zeng, S. Sun, J. Li, Z. L. Wang, and J. P. Liu, Appl. Phys. Lett. 85, 792 (2004).

${ }^{5}$ Y. Hou, S. Sun, C. Rong, and J. P. Liu, Appl. Phys. Lett. 91, 153117 (2007).

${ }^{6}$ C. B. Rong, V. Nandwana, N. Poudyal, J. P. Liu, M. E. Kozlov, R. H. Baughman, Y. Ding, and Z. L. Wang, J. Appl. Phys. 102, 023908 (2007).

${ }^{7}$ G. S. Chaubey, V. Nandwana, N. Poudyal, C. Rong, and J. P. Liu, Chem. Mater. 20, 475 (2008).

${ }^{8}$ C. B. Rong, V. Nandwana, N. Poudyal, J. P. Liu, T. Saito, Y. Q. Wu, and M. J. Kramer, J. Appl. Phys. 101, 09K515 (2007).

${ }^{9}$ C. B. Rong, V. Nandwana, N. Poudyal, Y. Li, J. P. Liu, Y. Ding, and Z. L. Wang, J. Phys. D 40, 712 (2007).

${ }^{10}$ D. Suess, T. Schrefl, S. Fähler, M. Kirschner, G. Hrkac, F. Dorfbauer, and J. Fidler, Appl. Phys. Lett. 87, 012504 (2005).

${ }^{11}$ V. Nandwana, K. E. Elkins, N. Poudyal, G. S. Chaubey, K. Yano, and J. P. Liu, J. Phys. Chem. C 111, 4185 (2007).

${ }^{12}$ B. D. Cullity, Introduction to Magnetic Materials (Addison-Wiley, London, 1972).

${ }^{13}$ H. Yu, M. Chen, P. M. Rice, S. X. Wang, R. L. White, and S. Sun, Nano Lett. 5, 379 (2005).

${ }^{14}$ C. B. Rong, Y. Z. Liu, and J. P. Liu, Appl. Phys. Lett. 93, 042508 (2008).

${ }^{15}$ C. B. Rong, H. W. Zhang, X. B. Du, J. Zhang, S. Y. Zhang, and B. G. Shen, J. Appl. Phys. 96, 3921 (2004).

${ }^{16}$ C. B. Rong, H. W. Zhang, R. J. Chen, S. L. He, and B. G. Shen, J. Magn. Magn. Mater. 302, 126 (2006)

${ }^{17}$ K. O'Grady, M. El-Hilo, and R. W. Chantrell, IEEE Trans. Magn. 29, 2608 (1993).

${ }^{18}$ C. B. Rong, H. W. Zhang, B. G. Shen, and J. P. Liu, Appl. Phys. Lett. 88, 042504 (2006). 